\title{
79. Datenschutzkonferenz in Stuttgart
}

Die Ergebnisse der 79. Konferenz der Datenschutzbeauftragten des Bundes und der Länder am 17./18. März 2010 in Stuttgart hat der diesjährige Konferenzvorsitzende, der baden-württembergische Datenschutzbeauftragte Jörg Klingbeil, gemeinsam mit dem Bundesbeauftragten für den Datenschutz und die Informationsfreiheit, Peter Schaar, und dem Berliner Beauftragten für Datenschutz und Informationsfreiheit, Dr. Alexander Dix, vorgestellt.

Die Datenschutzbeauftragten des Bundes und der Länder sehen sich durch das Urteil des Europäischen Gerichtshofs vom 09. März 2010 bestätigt, nach dem auch die deutschen Datenschutzaufsichtsbehörden von jeder Weisung durch Regierungsstellen völlig frei sein müssen, soweit sie private Datenverarbeiter kontrollieren. Sie halten daher eine grundsätzliche Neuordnung der Datenschutzaufsicht in Deutschland für geboten und fordern von den $\mathrm{Ge}$ setzgebern in Bund und Ländern eine europarechtlich einwandfreie Regelung, die für die Tätigkeit der unabhängigen Datenschutzbeauftragten im öffentlichen Bereich ebenfalls Folgen haben dürfte. Die in den Ländern und beim Bund bisher bestehenden Regelungen und Strukturen müssen im Einzelnen daraufhin überprüft werden, wie die Unabhängigkeit im Sinne des Urteils realisiert und im Interesse der Bürgerinnen und Bürger auch wirksam ausgestaltet werden kann. Eine Fach- und Rechtsaufsicht darf es nicht geben. ${ }^{1}$

Die Konferenz hat sich erneut mit der Modernisierung des Datenschutzrechts befasst. „Unser Recht passt nicht mehr ins Internetzeitalter", so Jörg Klingbeil. Als Grundlage für eine Diskussion über eine grundlegende Reform des Datenschutzrechts haben die Datenschutzbeauftragten ein Eckpunktepapier vorgelegt; u.a. werden darin ein technikneutraler Ansatz, die Stärkung der Betroffenenrechte und wirksamere Sanktionen gefordert (Zusammenfassung siehe ${ }^{2}$ ).

Das Bundesverfassungsgericht hat durch Urteil vom 02. März 2010 die Vorratsdatenspeicherung in der aktuellen

\footnotetext{
1 In diesem Heft: Dokumentation S. 329
}

2 In diesem Heft: Dokumentation S. 331
Form für verfassungswidrig erklärt. Die Datenschutzbeauftragten sehen sich durch die Entscheidung des höchsten deutschen Gerichts in ihrer Auffassung bestärkt. Die Konferenz lehnt die Vorratsdatenspeicherung grundsätzlich ab. Das Verbot der Totalerfassung gehöre zur verfassungsrechtlichen Identität der Bundesrepublik Deutschland. Die Datenschutzbeauftragten fordern die Bundesregierung auf, sich für eine Aufhebung der Europäischen Richtlinie 2006/24/EG einzusetzen. Nach Ansicht der Konferenz müsse die Entscheidung des Bundesverfassungsgerichts auch über die Kommunikationsdaten hinaus Beachtung finden, etwa bei Flugpassagierdaten oder bei der Konzeption von Mautsystemen. Auch die zentrale ELENA-Datenbank müsse auf den Prüfstand. ${ }^{3}$

Beim Einsatz von Ganzkörperscannern sieht die Konferenz der Datenschutzbeauftragten noch viele offene Fragen. Es müsse noch geklärt werden, was die Geräte technisch leisten können, ob damit ein nennenswerter Sicherheitsgewinn erzielbar ist und inwieweit Gesundheitsschäden entstehen können. Der Gesetzgeber habe nun über den Einsatz der Scanner zu entscheiden. Die Grundrechte der Betroffenen müssten dabei geschützt werden. Geschlechtsmerkmale oder künstliche Körperteile bzw. medizinische Hilfsmittel dürften nicht angezeigt werden. ${ }^{4}$

Für einige Sicherheitsgesetze der letzten Jahre ist eine Evaluierung vom Gesetzgeber vorgesehen worden. Allerdings ist eine wirksame Erfolgskontrolle entscheidend davon abhängig, unter welchen Vorgaben und von wem sie durchgeführt wird. Die Datenschutzbeauftragten des Bundes und der Länder sehen mit Sorge, dass bei der derzeit vorgesehenen Evaluierung des Gemeinsame-Dateien-Gesetzes externer wissenschaftlicher Sachverstand nur eine Alibifunktion haben könnte. Es sollte eigentlich eine Selbstverständlichkeit sein, dass der Gesetzgeber diese Erfolgskontrolle von unabhängigen Experten, die nicht der die Sicherheitsgesetze vollziehenden Gewalt angehören, durch-

\footnotetext{
3 In diesem Heft: Dokumentation S. 329
}

4 ebenda führen lässt. Dies gilt auch für die von der Bundesregierung vorgesehene Evaluierung der Kooperationszentren von Polizei und Nachrichtendiensten. ${ }^{5}$

Das Bundesgesundheitsministerium plant, die bisherige Regelung zur Abrechnung hausärztlicher Leistungen und ambulanter Notfallbehandlungen im Krankenhaus durch private Abrechnungsstellen in der gesetzlichen Krankenversicherung um ein Jahr zu verlängern. Damit setzt sich der Bundesgesundheitsminister nicht nur über ein Urteil des Bundessozialgerichts hinweg. Auch die Datenschutzbeauftragten halten die derzeitigen Regelungen zur Datenverarbeitung höchst sensibler Daten durch private Stellen für völlig unzureichend. Sie fordern daher vom Gesetzgeber, schnell präzise Vorgaben für die Einschaltung privater Stellen bei der Abrechnung von Kassenleistungen im Sozialgesetzbuch zu verankern. ${ }^{6}$

Das Bundesverfassungsgericht hat in seiner Entscheidung vom 20. Dezember 2007 eine unzulässige Mischverwaltung bei den JobCentern festgestellt. Dies führte zu unklaren Zuordnungen von Verantwortlichkeiten in den Arbeitsgemeinschaften beim sozialrechtlichen Datenschutz im Bereich des Arbeitslosengelds II. Die Konferenz der Datenschutzbeauftragten des Bundes und der Länder fordert deshalb für die anstehende Neuorganisation der Arbeitsgemeinschaften/JobCenter eindeutige gesetzliche Regelungen für die datenschutzrechtlichen Aufgaben und Kontrollzuständigkeiten, um die bisher bestehenden Unsicherheiten bei der Datenschutzkontrolle zu beseitigen. Keinesfalls dürfe es zu einer voraussetzungslosen Mehrfachspeicherung sensibler personenbezogener Daten bei den Leistungsträgern kommen, denn damit würden unvertretbare datenschutzrechtliche Risiken verbunden sein.

\section{Jörg Klingbeil \\ Landesbeauftragter für den Datenschutz, Baden-Württemberg}

\footnotetext{
5 ebenda

6 ebenda
} 